\title{
Quality of e-discharge summaries at a district general hospital
}

\author{
Authors: Mohummad S Goonoo, Ismail Al-Talib, Nidal Hammoud and Pankaj Chaturvedi
}

\section{Aims}

A standardised high-quality electronic discharge (e-discharge) summary ensures continuity of care of patients in the community. They are integral to bridging the gap between secondary and primary care by ensuring safe transfer of information. Poor communication can cause patient harm and significant incidents. The aim was to assess our local practice by reviewing e-discharge summaries, in order to ascertain whether we are achieving nationally agreed professional record keeping standards as defined by the Royal College of Physicians (RCP).

\section{Methods}

We collected data prospectively on 55 inpatients admitted to medical wards over a 2 -week period. We compared the documentation on the e-discharge summaries to the standards set by the RCP regarding recording of diagnosis, past medical history, do not attempt cardio-pulmonary resuscitation (DNACPR) decision, medication changes and reasons, general practitioner (GP) advice and follow up, hospital advice and follow-up and important investigations relevant to diagnosis.

\section{Results}

Of the 55 patients ( 17 males, 38 females; median age 77.5 years) considered, the discharge summaries were completed by junior doctors in $78 \%$ of cases. Twenty-five per cent had no diagnosis documented for the current admission episode; $42 \%$ had no documentation of past medical history; $60 \%$ had an escalation plan including DNACPR but this was not included in the discharges. About $75 \%$ of patients had a clear follow-up note written and communicated to their GP. Forty-nine patients had documentation of medication changes, out of which only 33 had the reasons recorded.

\section{Conclusion}

Our e-discharge summaries are not fully compliant with RCP recommendations, potentially widening the communication gap to GPs. We have adopted the RCP's recommendations, designed a discharge summary template, which has been disseminated for use across the trust and we plan to review its impact on adherence to guidelines.

\section{Conflict of interest statement}

None declared. 